\title{
Knee pain as a predictor of structural progression over 4 years: data from the Osteoarthritis Initiative, a prospective cohort study
}

Yuanyuan Wang ${ }^{1 *} \mathbb{D}$, Andrew J. Teichtahl', François Abram², Sultana Monira Hussain', Jean-Pierre Pelletier ${ }^{3}$, Flavia M. Cicuttini ${ }^{1+}$ and Johanne Martel-Pelletier ${ }^{3+}$

\begin{abstract}
Background: There is evidence that knee pain not only is a consequence of structural deterioration in osteoarthritis $(\mathrm{OA})$ but also contributes to structural progression. Clarifying this is important because targeting the factors related to knee pain may offer a clinical approach for slowing the progression of knee OA. The aim of this study was to examine whether knee pain over 1 year predicted cartilage volume loss, incidence and progression of radiographic osteoarthritis (ROA) over 4 years.

Methods: Osteoarthritis Initiative participants with no ROA (Kellgren-Lawrence grade $\leq 1)(n=2120)$ and with ROA (Kellgren-Lawrence grade $\geq 2)(n=2249)$ were examined. Knee pain was assessed at baseline and 1 year using the Western Ontario and McMaster Universities Osteoarthritis Index (WOMAC). Knee pain patterns were categorised as no pain (WOMAC pain $<5$ at baseline and 1 year), fluctuating pain (WOMAC pain $\geq 5$ at either time point) and persistent pain (WOMAC pain $\geq 5$ at both time points). Cartilage volume, incidence and progression of $\mathrm{ROA}$ were assessed using magnetic resonance imaging and $\mathrm{x}$-rays at baseline and 4-years.
\end{abstract}

Results: In both non-ROA and ROA, greater baseline WOMAC knee pain score was associated with increased medial and lateral cartilage volume loss ( $p \leq 0.001$ ), incidence (OR 1.07, 95\% Cl 1.01-1.13) and progression (OR $1.07,95 \% \mathrm{Cl} 1.03-1.10)$ of ROA. Non-ROA and ROA participants with fluctuating and persistent knee pain had increased cartilage volume loss compared with those with no pain ( $p$ for trend $\leq 0.01$ ). Non-ROA participants with fluctuating knee pain had increased risk of incident ROA (OR 1.62,95\% Cl 1.04-2.54), corresponding to a number needed to harm of 19.5. In ROA the risk of progressive ROA increased in participants with persistent knee pain (OR 1.82, 95\% Cl 1.28-2.60), corresponding to a number needed to harm of 9.6.

Conclusions: Knee pain over 1 year predicted accelerated cartilage volume loss and increased risk of incident and progressive ROA. Early management of knee pain and controlling knee pain over time by targeting the underlying mechanisms may be important for preserving knee structure and reducing the burden of knee OA.

Keywords: Pain, Knee osteoarthritis, Cartilage, Incidence, Progression, Magnetic resonance imaging

\footnotetext{
* Correspondence: yuanyuan.wang@monash.edu

${ }^{\dagger}$ Flavia M. Cicuttini and Johanne Martel-Pelletier contributed equally to this work.

'Department of Epidemiology and Preventive Medicine, School of Public

Health and Preventive Medicine, Monash University, 553 St Kilda Road,

Melbourne, VIC 3004, Australia

Full list of author information is available at the end of the article
}

(c) The Author(s). 2018 Open Access This article is distributed under the terms of the Creative Commons Attribution 4.0 International License (http://creativecommons.org/licenses/by/4.0/), which permits unrestricted use, distribution, and reproduction in any medium, provided you give appropriate credit to the original author(s) and the source, provide a link to the Creative Commons license, and indicate if changes were made. The Creative Commons Public Domain Dedication waiver (http://creativecommons.org/publicdomain/zero/1.0/) applies to the data made available in this article, unless otherwise stated. 


\section{Background}

Pain and structural articular degeneration are major clinical manifestations of knee osteoarthritis (OA). Although previous studies have predominantly focussed on whether structural disease progression predicts knee pain in people with knee OA [1-4], there have been relatively few studies examining whether knee pain is a predictor of structural progression of knee OA $[5,6]$. There is increasing evidence for an important interplay between joint structures such as cartilage, bone, muscle and other soft tissues in maintaining joint health [7]. Pain through mechanisms such as inflammation and reduced mobility can adversely affect these joint structures, resulting in structural progression $[8,9]$. Thus it is plausible that knee pain not only is a consequence of structural deterioration in $\mathrm{OA}$ but also contributes to structural progression. Clarifying this is important, because if this is the case, targeting the factors related to knee pain may offer a potential strategy for slowing disease progression of $\mathrm{OA}$.

The major structural outcomes commonly examined in the development and progression of knee OA include cartilage volume loss assessed by magnetic resonance imaging (MRI) and incidence and progression of radiographic osteoarthritis (ROA). The findings of prospective cohort studies examining whether knee pain is a predictor of structural progression are summarised in Table 1. Inconsistent results have emerged regarding whether knee pain predicts cartilage volume/thickness loss $[1,5,10-14]$. This may be attributable to small to moderate sample sizes, different study populations, subgroup analyses and varied outcome measures. Although some studies found no association between baseline knee pain and subsequent cartilage volume loss in symptomatic knee OA $[1,10,13]$ or asymptomatic [11] individuals, other studies showed relationships of baseline knee pain [14], frequent knee pain [5] and change in knee pain $[1,12,13]$ with cartilage volume/ thickness loss. In terms of studies with radiographic outcomes, some studies have suggested no association between baseline knee pain and progression of ROA [6, 15-17], whereas other studies have reported associations of baseline knee pain with incident ROA [16, 18], incident accelerated knee OA [19] and progressive ROA [18]. Differences in study population, assessment of knee pain, duration of follow-up and definition of incidence and progression of knee ROA may provide potential explanations of the inconclusive results. Larger cohort studies with longer follow-up have shown significant associations between knee pain and incidence and/or progression of knee ROA [16, 18, 19].

The National Institutes of Health Osteoarthritis Initiative (OAI) is the largest observational cohort of knee OA [20] and offers the opportunity to examine whether knee pain predicts structural progression. The aim of the present study was to examine whether baseline knee pain and knee pain patterns over 1 year are predictors of cartilage volume loss, incidence and progression of ROA over 4 years in a large cohort of individuals with and without knee ROA.

\section{Methods \\ Osteoarthritis Initiative}

Data were extracted from the OAI database, which holds data derived from a publicly available, multicentre, population-based cohort study of knee OA (https:// oai.nih.gov). The OAI comprises data of 4796 participants aged 45-79 years with or at risk for knee OA at baseline. OAI exclusion criteria were inflammatory arthritis, severe joint space narrowing in both knees, unilateral knee replacement and severe joint space narrowing in the contralateral knee, inability to undergo MRI or provide a blood sample, use of walking aids except a single straight cane $\leq 50 \%$ of the time, or unwillingness to provide informed consent. Participants were recruited at four clinical sites, and the study was approved by the institutional review board at each of the sites. All participants gave informed consent.

\section{Participants of the current study}

Bilateral standing posteroanterior fixed-flexion knee radiographs [21] were assessed for baseline KellgrenLawrence $(\mathrm{K}-\mathrm{L})$ grading $(0-4)(n=4369)$. If both knees had no evidence of ROA, the dominant knee was selected for analyses. If only one knee had evidence of ROA, this was the selected knee for analyses. If both knees had evidence of ROA, the most severe knee was selected for analyses. When the severity was equal between sides, the most painful knee was selected for analyses. In the case of equal pain in both knees, the dominant knee was selected for analyses. Participants had been categorized into two groups based on their baseline K-L grade as part of their participation in the study: non-ROA (incidence cohort) defined by a baseline K-L grade $\leq 1 \quad(n=2120)$ and ROA (progression cohort) defined by a baseline K-L grade $\geq 2(n=2249)$.

\section{Knee pain assessment}

Knee pain was assessed yearly using the Western Ontario and McMaster Universities Osteoarthritis Index (WOMAC) pain subscale [22], Likert scale version. It consists of five items with scores ranging 0 to 20 and 20 being the worst pain. "Symptomatic" was defined as a WOMAC pain score $\geq 5$ based on the Low-Intensity Symptom State-Attainment Index cut-off [23]. This definition has been used in a previous OAI study in which WOMAC knee pain score $\geq 5$ represented the upper tertile of all participants with any pain in the 


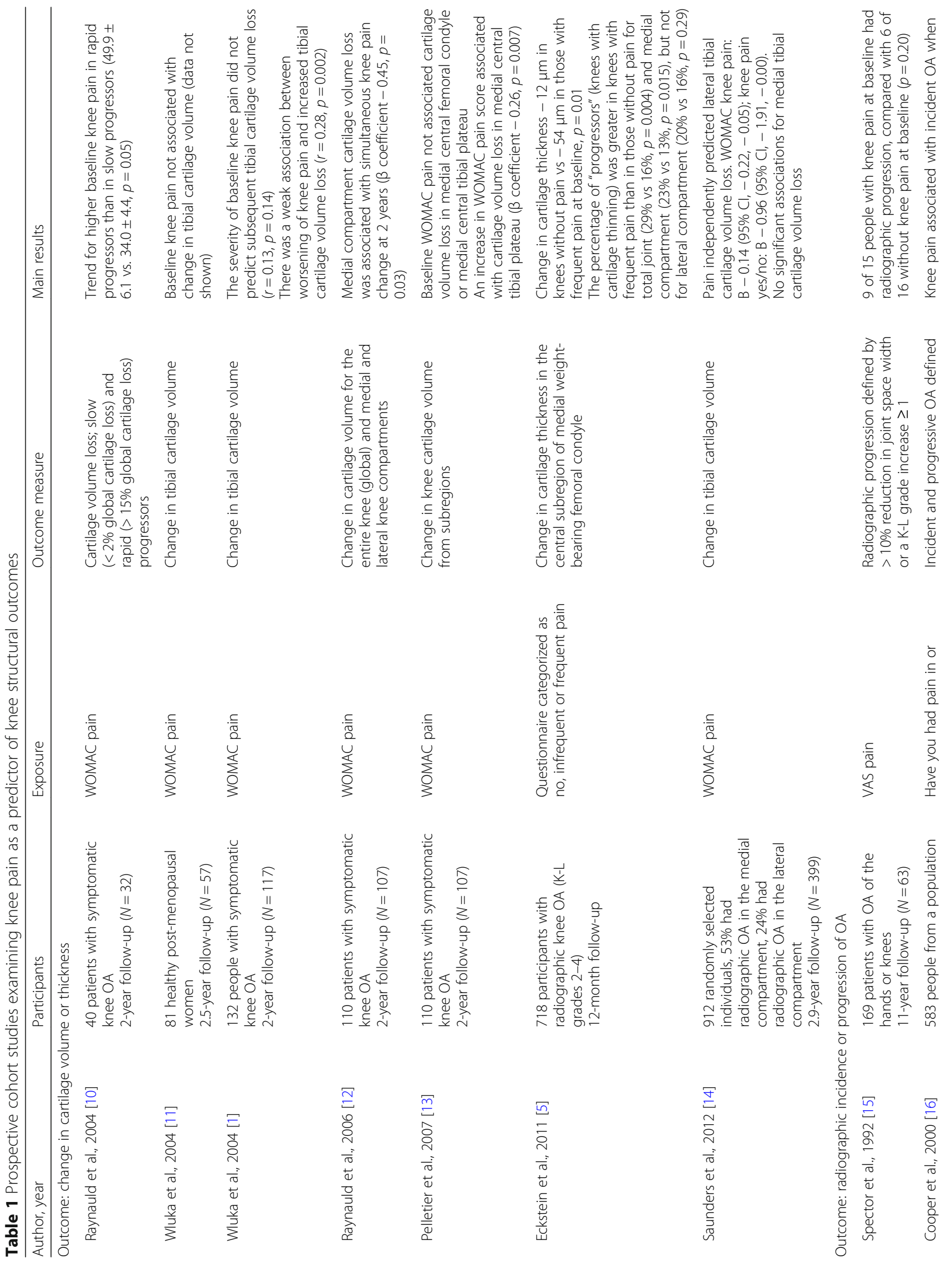




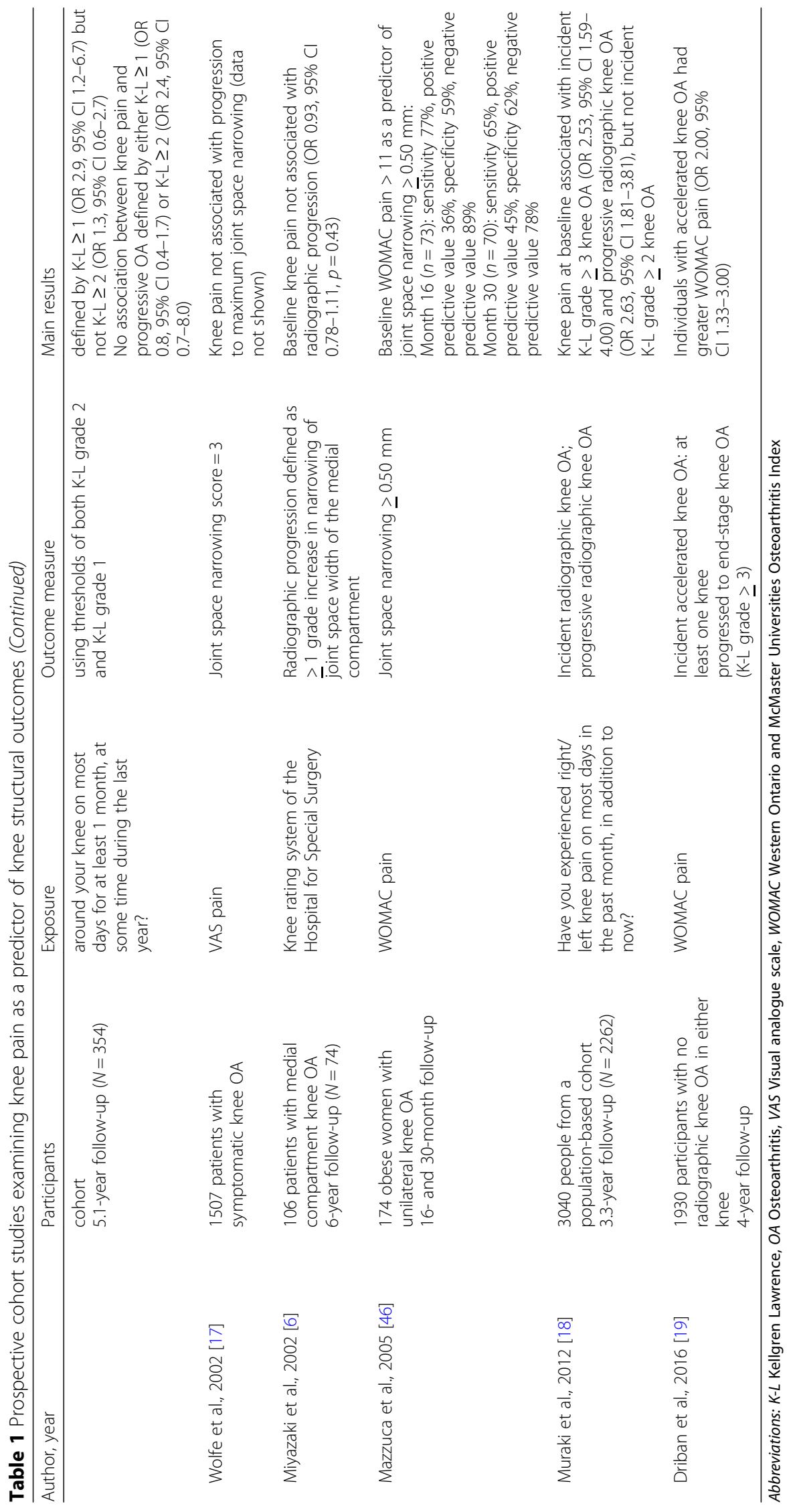


cohort [24]. The knee pain patterns from baseline to 1-year follow-up were categorised as follows: no knee pain (WOMAC pain $<5$ at both baseline and 1 year), fluctuating knee pain (WOMAC pain $\geq 5$ at either baseline or 1 year) and persistent knee pain (WOMAC pain $\geq 5$ at both baseline and 1 year).

\section{Cartilage volume assessment}

Knee MRI was performed for the target knee using a 3-T apparatus (Magnetom Trio; Siemens, Erlangen, Germany). Cartilage volume was measured by sagittal double-echo steady-state imaging for medial and lateral tibiofemoral compartments (condyle and plateau) using an automatic human cartilage segmentation (ArthroLab, Montreal, QC, Canada) as previously described and validated $[25,26]$. The test-retest revealed an excellent measurement error of $0.3 \pm 1.6 \%$, corresponding to a measurement error of $30.3 \pm$ $126.2 \mathrm{~mm}^{3}$ [26]. The annual rate of cartilage volume loss over 4 years was obtained by calculating (4-year follow-up volume - baseline volume)/baseline volume/ 4 , expressed as a percentage.

\section{Assessment of incidence and progression of ROA}

Incidence of ROA was defined by a baseline $\mathrm{K}-\mathrm{L}$ grade of 0 or 1 and a K-L grade $\geq 2$ at 4-year follow-up. Progression of ROA was defined by a baseline K-L grade of 2 or 3 and an increase in K-L grade $\geq 1$ at 4 -year follow-up.

\section{Statistical analyses}

Demographic, clinical, radiological and MRI data were systematically entered into a computerized database. Participant characteristics were compared between participants with and without ROA using independent samples $t$ tests or chi-square tests when appropriate. With 2120 non-ROA participants, our study had $80 \%$ power to detect a regression coefficient as low as 0.006 between baseline knee pain and cartilage volume loss with five predictors, and a relative risk as low as 1.41 between baseline knee pain and incidence of ROA, $\alpha$ error of 0.05 , two-sided significance. With 2249 participants with ROA, our study had $80 \%$ power to detect a regression coefficient as low as 0.0057 between baseline knee pain and cartilage volume loss with five predictors, and a relative risk as low as 1.27 between baseline knee pain and progression of ROA, $\alpha$ error of 0.05 , two-sided significance. The association between baseline knee pain and cartilage volume loss was examined using multiple linear regression. The association between knee pain patterns over 1 year and cartilage volume loss was examined using the $F$-test (generalised linear model) with estimated marginal means (SE), and linear trend was assessed using multiple linear regression. The associations of baseline knee pain and knee pain patterns over 1 year with incidence and progression of ROA were examined using binary logistic regression. The attributable risk and number needed to harm $(\mathrm{NNH})$ were calculated. $\mathrm{NNH}$ is a measure of how many people need to be exposed to a risk factor in order for one person to have a particular adverse effect. All the analyses were adjusted for gender, baseline age, body mass index (BMI) and K-L grade. All tests were two-sided, and $p<0.05$ was considered statistically significant. Statistical analyses were performed using the IBM SPSS Statistics software package (version 24; IBM, Armonk, NY, USA).

\section{Results}

Participant characteristics at baseline, as well as knee pain and structure changes over time, are shown in Table 2. Compared with non-ROA participants, participants with ROA were older, had higher BMIs and WOMAC pain scores, were more likely to have fluctuating and persistent knee pain, and had a greater rate of cartilage volume loss (all $p<0.001$ ). The incidence and progression of ROA were $9.6 \%$ and $17.8 \%$, respectively. There was no significant difference in age, gender or BMI between those who completed $(n=3395)$ and those who did not complete $(n=974)$ the 4-year follow-up. The non-completers had higher K-L grade and worse knee pain and were more likely to have ROA than the completers (all $p<0.05$ ).

\section{Associations between baseline knee pain and structural progression over 4 years}

In non-ROA, a greater baseline WOMAC pain score was associated with increased rate of cartilage volume loss in medial (regression coefficient $0.04 \%$, 95\% CI $0.02-0.06 \%)$ and lateral $(0.04 \%, 0.02-0.06 \%)$ compartments by MRI, adjusted for age, gender, BMI and K-L grade. A higher baseline WOMAC pain score was also associated with increased incidence of ROA (OR 1.07, 95\% CI 1.01-1.13) (top half of Table 3). In ROA, a greater baseline WOMAC pain score was associated with increased rate of cartilage volume loss in medial (regression coefficient $0.04 \%, 95 \% \mathrm{CI} 0.02-0.07 \%$ ) and lateral $(0.05 \%, 0.03-0.07 \%)$ compartments by MRI. A higher baseline WOMAC pain score was also associated with increased progression of ROA (OR 1.07, 95\% CI 1.03-1.10) (bottom half of Table 3).

\section{Associations between knee pain patterns over 1 year and structural progression over 4 years}

In non-ROA, the annual rate of cartilage volume loss in the medial compartment was $0.63 \%$ (SE $0.03 \%$ ) in participants with no knee pain, $0.81 \%(0.08 \%)$ in those with fluctuating knee pain, and $0.93 \%(0.12 \%)$ in those with 
Table 2 Characteristics of study participants

\begin{tabular}{|c|c|c|c|}
\hline & $\begin{array}{l}\text { Non-ROA } \\
n=2120\end{array}$ & $\begin{array}{l}\text { ROA } \\
n=2249\end{array}$ & $p$ Value \\
\hline \multicolumn{4}{|l|}{ Baseline characteristics } \\
\hline Age, years & $59.9(9.1)$ & $62.7(8.9)$ & $<0.001$ \\
\hline Female, $n(\%)$ & $1224(57.7)$ & $1311(58.3)$ & 0.71 \\
\hline Body mass index, $\mathrm{kg} / \mathrm{m}^{2}$ & $27.6(4.5)$ & $29.8(4.8)$ & $<0.001$ \\
\hline Kellgren-Lawrence grade, $n(\%)$ & & & - \\
\hline 0 & $1432(67.5)$ & - & \\
\hline 1 & $688(32.5)$ & - & \\
\hline 2 & - & $1173(52.2)$ & \\
\hline 3 & - & $787(35.0)$ & \\
\hline 4 & - & $289(12.8)$ & \\
\hline WOMAC pain score (range $0-20$ ) & $1.9(2.7)$ & $3.7(3.8)$ & $<0.001$ \\
\hline WOMAC pain score $\geq 5, n(\%)$ & $307(14.5)$ & $760(33.8)$ & $<0.001$ \\
\hline \multicolumn{4}{|l|}{ Change in knee pain and structure } \\
\hline Knee pain pattern over 1 year, $n(\%)$ & & & $<0.001$ \\
\hline No knee pain at both baseline and 1 year & $1669(80.5)$ & $1265(57.8)$ & \\
\hline Fluctuating knee pain (pain at either baseline or 1 year) & $274(13.2)$ & $464(21.2)$ & \\
\hline Persistent knee pain (pain at both baseline and 1 year) & $131(6.3)$ & $459(21.0)$ & \\
\hline \multicolumn{4}{|l|}{ Annual percentage cartilage volume loss over 4 years } \\
\hline Medial compartment & $0.68(1.13)$ & $1.36(1.95)$ & $<0.001$ \\
\hline Lateral compartment & $0.73(1.03)$ & $1.21(1.41)$ & $<0.001$ \\
\hline Incidence of ROA over 4 years, $n(\%)$ & $165(9.6)$ & - & - \\
\hline Progression of ROA over 4 years, $n(\%)$ & - & $272(17.8)$ & - \\
\hline
\end{tabular}

ROA Radiographic osteoarthritis, WOMAC Western Ontario and McMaster Universities Osteoarthritis Index Data displayed as mean (SD) or number (\%)

Table 3 Associations of baseline Western Ontario and McMaster Universities Osteoarthritis Index knee pain score with annual percentage cartilage volume loss and incidence and progression of radiographic knee osteoarthritis over 4 years

\begin{tabular}{|c|c|c|c|c|}
\hline & Univariable analysis & & Multivariable analysis $^{a}$ & \\
\hline \multicolumn{5}{|l|}{ Non-ROA } \\
\hline & Regression coefficient $(95 \% \mathrm{Cl})$ & $p$ Value & Regression coefficient $(95 \% \mathrm{Cl})$ & $p$ Value \\
\hline Annual percentage cartilage volume loss in medial compartment & $0.04(0.01,0.06)$ & 0.001 & $0.04(0.02,0.06)$ & 0.001 \\
\hline \multirow[t]{2}{*}{ Annual percentage cartilage volume loss in lateral compartment } & $0.04(0.02,0.06)$ & $<0.001$ & $0.04(0.02,0.06)$ & $<0.001$ \\
\hline & OR $(95 \% \mathrm{Cl})$ & $p$ Value & OR $(95 \% \mathrm{Cl})$ & $p$ Value \\
\hline Incidence of radiographic knee osteoarthritis & $1.10(1.05,1.16)$ & $<0.001$ & $1.07(1.01,1.13)$ & 0.02 \\
\hline \multicolumn{5}{|l|}{$\mathrm{ROA}$} \\
\hline & Regression coefficient $(95 \% \mathrm{Cl})$ & $p$ Value & Regression coefficient $(95 \% \mathrm{Cl})$ & $p$ Value \\
\hline Annual percentage cartilage volume loss in medial compartment & $0.06(0.03,0.08)$ & $<0.001$ & $0.04(0.02,0.07)$ & 0.001 \\
\hline \multirow[t]{2}{*}{ Annual percentage cartilage volume loss in lateral compartment } & $0.06(0.04,0.08)$ & $<0.001$ & $0.05(0.03,0.07)$ & $<0.001$ \\
\hline & OR $(95 \% \mathrm{Cl})$ & $p$ Value & OR $(95 \% \mathrm{Cl})$ & $p$ Value \\
\hline Progression of radiographic knee osteoarthritis & $1.08(1.05,1.12)$ & $<0.001$ & $1.07(1.03,1.10)$ & $<0.001$ \\
\hline
\end{tabular}


persistent knee pain ( $p$ for trend $=0.003$ ), adjusted for age, gender, BMI, and K-L grade (Table 4). The rate was greater in participants with fluctuating $(p=0.04)$ and persistent $(p=0.02)$ knee pain than in those without knee pain. Similar results were found in the lateral compartment. Although persistent knee pain was not significantly associated with the incidence of ROA (OR 1.57, 95\% CI 0.85-2.90), fluctuating knee pain was associated with increased incidence of ROA (OR 1.62, 95\% CI 1.04-2.54; $p$ for trend $=0.03$ ) (Table 4). The attributable risk of fluctuating knee pain for incident ROA was 35\% (4-55\%), with an NNH of 19.5 .

In ROA, the annual rate of cartilage volume loss in the medial compartment was $1.26 \%$ (SE $0.06 \%$ ) in participants with no knee pain, $1.47 \%(0.11 \%)$ in those with fluctuating knee pain, and $1.60 \%(0.12 \%)$ in those with persistent knee pain ( $p$ for trend $=0.01$ ), adjusted for age, gender, BMI and K-L grade (Table 5). The rate was greater in participants with persistent $(p=0.02)$ knee pain than in those without knee pain. Similar results were shown in the lateral compartment. Although fluctuating knee pain was not significantly associated with the progression of ROA (OR 1.34, 95\% CI 0.94-1.89), persistent knee pain was associated with increased progression of ROA (OR 1.82, 95\% CI 1.28-2.60; $p$ for trend $=0.001)$ (Table 5). The attributable risk of persistent knee pain for progressive ROA was 37\% (18-50\%), corresponding to the $\mathrm{NNH}$ of 9.6.

Similar results were observed for the association between knee pain patterns over 2 and 3 years and structural progression over 4 years (Additional file 1: Tables S1 and S2).

\section{Discussion}

In this large prospective cohort study, greater baseline knee pain, as well as fluctuating and persistent knee pain over 1 year, predicted structural progression over 4 years in participants with and without knee ROA, as evidenced by accelerated cartilage volume loss and increased incidence and progression of ROA. Among non-ROA participants with fluctuating knee pain over 1 year, $35 \%$ of the incident ROA risk over 4 years could be attributed to fluctuating knee pain, corresponding to an $\mathrm{NNH}$ of 19.5. In participants with ROA with persistent knee pain over 1 year, 37\% of the progressive ROA risk over 4 years could be attributed to persistent knee pain, corresponding to an $\mathrm{NNH}$ of 9.6. These data suggest that knee pain is an important predictive factor for the deterioration of knee structural outcomes and highlight the significant adverse impact of persistent knee

Table 4 Associations of knee pain patterns over 1 year with annual percentage cartilage volume loss and incidence of radiographic knee osteoarthritis over 4 years in participants without radiographic knee osteoarthritis at baseline

\begin{tabular}{|c|c|c|c|c|}
\hline & \multicolumn{2}{|l|}{ Univariable analysis } & \multicolumn{2}{|l|}{ Multivariable analysis $^{a}$} \\
\hline & Estimated marginal mean (SE) & $p$ Value & Estimated marginal mean (SE) & $p$ Value \\
\hline \multicolumn{5}{|c|}{ Annual percentage cartilage volume loss in medial compartment } \\
\hline No knee pain at both baseline and 1 year & $0.63(0.03)$ & & $0.63(0.03)^{b, c}$ & \\
\hline Fluctuating knee pain (pain at either time point) & $0.80(0.08)$ & $0.01^{\mathrm{d}}$ & $0.81(0.08)^{b}$ & $0.01^{\mathrm{d}}$ \\
\hline Persistent knee pain (pain at both time points) & $0.94(0.12)$ & & $0.93(0.12)^{c}$ & \\
\hline Trend & & 0.004 & & 0.003 \\
\hline \multicolumn{5}{|c|}{ Annual percentage cartilage volume loss in lateral compartment } \\
\hline No knee pain at both baseline and 1 year & $0.68(0.03)$ & & $0.68(0.03)^{e, f}$ & \\
\hline Fluctuating knee pain (pain at either time point) & $0.88(0.08)$ & $0.008^{d}$ & $0.89(0.08)^{\mathrm{e}}$ & $0.005^{\mathrm{d}}$ \\
\hline Persistent knee pain (pain at both time points) & $0.92(0.11)$ & & $0.93(0.11)^{f}$ & \\
\hline \multirow[t]{2}{*}{ Trend } & & 0.003 & & 0.002 \\
\hline & OR $(95 \% \mathrm{Cl})$ & $p$ Value & OR $(95 \% \mathrm{Cl})$ & $p$ Value \\
\hline \multicolumn{5}{|l|}{ Incidence of radiographic knee osteoarthritis } \\
\hline No knee pain at both baseline and 1 year & 1.00 & & 1.00 & \\
\hline Fluctuating knee pain (pain at either time point) & $1.96(1.29,2.99)$ & 0.002 & $1.62(1.04,2.54)$ & 0.03 \\
\hline Persistent knee pain (pain at both time points) & $2.07(1.16,3.72)$ & 0.01 & $1.57(0.85,2.90)$ & 0.15 \\
\hline Trend & & $<0.001$ & & 0.03 \\
\hline
\end{tabular}

${ }^{a}$ Adjusted for age, gender, body mass index and Kellgren-Lawrence grade

${ }^{\mathrm{b}} p=0.04$ for between-group difference

${ }^{c} p=0.02$ for between-group difference

${ }^{d}$ For difference in annual percentage cartilage volume loss in medial/lateral compartment among the three knee pain pattern groups

${ }^{\mathrm{e}} p=0.01$ for between-group difference

${ }^{f} p=0.03$ for between-group difference 
Table 5 Associations of knee pain patterns over 1 year with annual percentage cartilage volume loss and progression of radiographic knee osteoarthritis over 4 years in participants with radiographic knee osteoarthritis at baseline

\begin{tabular}{|c|c|c|c|c|}
\hline & \multicolumn{2}{|l|}{ Univariable analysis } & \multicolumn{2}{|l|}{ Multivariable analysis $^{a}$} \\
\hline & Estimated marginal mean (SE) & $\overline{p \text { Value }}$ & Estimated marginal mean (SE) & $p$ Value \\
\hline \multicolumn{5}{|c|}{ Annual percentage cartilage volume loss in medial compartment } \\
\hline No knee pain at both baseline and 1 year & $1.22(0.06)$ & & $1.26(0.06)^{\mathrm{b}}$ & \\
\hline Fluctuating knee pain (pain at either time point) & $1.52(0.11)$ & $0.001^{c}$ & $1.47(0.11)$ & $0.03^{c}$ \\
\hline Persistent knee pain (pain at both time points) & $1.68(0.12)$ & & $1.60(0.12)^{b}$ & \\
\hline Trend & & $<0.001$ & & 0.01 \\
\hline \multicolumn{5}{|c|}{ Annual percentage cartilage volume loss in lateral compartment } \\
\hline No knee pain at both baseline and 1 year & $1.06(0.05)$ & & $1.08(0.05)^{\mathrm{d}, \mathrm{e}}$ & \\
\hline Fluctuating knee pain (pain at either time point) & $1.41(0.08)$ & $<0.001^{\mathrm{c}}$ & $1.38(0.08)^{d}$ & $<0.001^{c}$ \\
\hline Persistent knee pain (pain at both time points) & $1.46(0.09)$ & & $1.39(0.09)^{\mathrm{e}}$ & \\
\hline \multirow[t]{2}{*}{ Trend } & & $<0.001$ & & $<0.001$ \\
\hline & Odds ratio $(95 \% \mathrm{Cl})$ & $p$ Value & Odds ratio $(95 \% \mathrm{Cl})$ & $p$ Value \\
\hline \multicolumn{5}{|l|}{ Progression of radiographic knee osteoarthritis } \\
\hline No knee pain at both baseline and 1 year & 1.00 & & 1.00 & \\
\hline Fluctuating knee pain (pain at either time point) & $1.46(1.04,2.06)$ & 0.03 & $1.34(0.94,1.89)$ & 0.10 \\
\hline Persistent knee pain (pain at both time points) & $2.13(1.53,2.97)$ & $<0.001$ & $1.82(1.28,2.60)$ & 0.001 \\
\hline Trend & & $<0.001$ & & 0.001 \\
\hline
\end{tabular}

*Adjusted for age, gender, body mass index and Kellgren-Lawrence grade

${ }^{\mathrm{b}} p=0.02$ for between-group difference

' For difference in annual percentage cartilage volume loss in medial/lateral compartment among the three knee pain pattern groups

${ }^{\mathrm{d}} p=0.001$ for between-group difference

${ }^{\mathrm{e}} p=0.002$ for between-group difference

pain on knee structures. The findings suggest that treating patients with knee pain both early in the disease course and over time is important for preserving knee structure and is likely to have a significant impact on reducing disease burden.

There have been conflicting data on the association between knee pain and structural progression of knee OA $[1,5,6,10,12-19]$. This may have resulted from limited sample sizes and durations of follow-up in both MRI and radiological studies. Cohort studies with larger sample sizes and/or longer durations of follow-up have shown significant associations of knee pain with MRI [5, 14] and radiographic [16, 18, 19] outcomes. One cohort study analysing baseline and 12-month follow-up data in a large subsample of 718 participants with K-L grades 2-4 from the OAI showed knees with frequent pain had greater rates of cartilage thickness loss in the central subregion of the medial femoral condyle than knees without pain [5]. Our study of a large knee ROA cohort with 4 years of follow-up showed that higher levels of baseline knee pain predicted increased structural progression over 4 years assessed by both MRI (cartilage volume loss) and $\mathrm{x}$-ray (progression of ROA). Our study extended the previous OAI study [5] by examining baseline knee pain as a continuous variable, thus indicating a dose-response relationship; by investigating knee pain pattern over time and its association with structural outcomes, showing associations for cartilage volume loss of both medial and lateral tibiofemoral compartments and consistent results for MRI and radiographic outcomes; and by examining participants with and without knee ROA simultaneously. The effect sizes for some of the associations appeared small, particularly for those between baseline WOMAC knee pain score and annual percentage cartilage volume loss (Table 3). However, it is important to put them in context. For example, in those with no ROA, even small changes in the annual rate of cartilage volume loss for small changes in knee pain will have significant impacts over many years. Only three studies have examined the relationship between knee pain and structural changes in non-OA populations $[11,16,19]$. Although one study found no association between baseline knee pain and tibial cartilage volume loss over 2.5 years [11], the other reported baseline knee pain being associated with incident ROA defined by $\mathrm{K}-\mathrm{L} \geq 1$ but not $\mathrm{K}-\mathrm{L} \geq 2$ over 5.1 years [16] and incident accelerated knee OA over 4 years [19]. We defined incident ROA using the more stringent K-L grade $\geq 2$ at follow-up. Our large 4-year follow-up study of participants with no ROA demonstrated that baseline knee pain predicted cartilage volume loss and 
incidence of ROA, suggesting a predictive role of knee pain in adverse structural outcomes, even in people without knee ROA.

Given the fluctuating nature of knee pain, we examined the association between knee pain patterns over 1 year and structural progression over 4 years. We found that fluctuating and persistent knee pain over 1 year predicted increased cartilage volume loss as well as incidence and progression of ROA over 4 years in participants with and without ROA, with positive linear relationships observed between the frequency of knee pain over 1 year and structural progression over 4 years. Previous studies reported that worsening of knee pain was associated with increased cartilage volume loss simultaneously (i.e., over the same time period) $[1,12,13]$. The adverse effect of ongoing (fluctuating or persistent) knee pain on knee structure has not previously been examined. We found that fluctuating and persistent knee pain over 1 year contributed substantially to the incidence and progression of ROA over 4 years. For every 20 non-ROA participants with fluctuating knee pain over 1 year, 1 developed incident ROA in 4 years. For every ten ROA participants with persistent knee pain over 1 year, one had progressive ROA in 4 years. These findings support the importance of controlling knee pain over time and that targeting people with fluctuating or persistent knee pain for early intervention will be important for preserving knee structure and delaying structural progression.

The mechanism of knee pain and structural change is likely to be multifactorial. Optimal knee function requires a complex interplay of structural and biomechanical factors, including supporting musculature. It has been shown that increased size and decreased fat content of the vastus medialis predict reduced cartilage volume loss, and the most significant predictor of increased muscle size is an improvement in knee pain [27-29]. Nonetheless, the causes of knee pain are complex and heterogeneous and include structural factors [30], inflammatory hyperalgesia [31], central mechanisms incorporating brain areas processing fear, emotions and in aversive conditioning [32], and genetic predispositions toward peripheral pain sensitisation [33]. There is evidence that inflammation $[8,9]$ and other structural abnormalities, such as bone marrow lesions and effusion-synovitis [34-36], are associated with greater knee pain and structural progression. Greater K-L grade is associated with higher levels of knee pain [30] and accelerated cartilage loss [37]. However, it is less likely that our findings are explained by disease severity, because all the analyses were adjusted for baseline K-L grade, and the outcome of annual percentage loss in cartilage volume took into account the baseline amount of cartilage volume. Understanding and appropriately targeting the factors related to knee pain in an individual may be particularly important for modifying disease trajectory, such as targeting quadriceps strengthening, bone marrow lesions, synovitis, central sensitisation, or weight loss.

This study has limitations. The selection of target knee for each participant was based on radiographic severity. It is more likely that this target knee is the one contributing to pain, but it is also possible that knee pain originates from the other knee and that there has been switching. This would have underestimated the magnitude of observed associations. There is also evidence that pain in one knee has adverse effects on the other knee through compensatory gait mechanisms that shift the load distribution from the affected limb to the healthy contralateral limb during weight-bearing activities [38, 39]. We did not adjust our results for medications that may influence pain over time, because the information was obtained by questionnaire, and the answers were limited. However, any symptoms experienced by the participants, including fluctuating symptoms, would have been present despite any therapies, so the notion of pain predicting structural progression remains valid. Furthermore, knee pain was analysed prior to the assessment of structural outcomes, thus it is unlikely that these results can be explained simply by structural changes causing pain. Although there was potential selection bias in the study, in which the non-completers had higher K-L grade and worse knee pain than the completers, this would not affect the interpretation of our results. We did not examine other structural abnormalities associated with pain, such as bone marrow lesions and effusion-synovitis [40-42], but this does not affect the interpretation of our findings, because such structural alterations are likely to be on the causal pathway from knee pain to structural outcomes [43-45]. There was an issue of multiple testing in our analyses. For each of the incidence cohort and the progression cohort, we examined two knee pain variables and three outcome variables, and thus we performed six tests. If we performed the Bonferroni correction, the significance level should be $0.05 / 6=0.008$. Most of our results remained significant after applying the Bonferroni correction. However, it is important to consider that we did not perform completely independent, unrelated analyses, because both cartilage volume loss and radiographic changes are on the same disease pathway. We found consistent results for incidence and progression of ROA, as well as for MRI and radiographic outcomes, after adjustment for potential confounders, suggesting a true association rather than a chance association. There is also the possibility of residual confounding that knee pain could be a biomarker of other unmeasured factors which are associated with structural progression. 
The present study has several strengths. The OAI offered a unique opportunity to study the disease profile of a large number of participants and explore the impact of knee pain on structural progression. Until now, the assessment of cartilage volume change by quantitative MRI has mostly been done using manual or semi-automated technologies, which have the intrinsic limitation of variability in results with respect to human intervention. This imposed limitations on a complete analysis of the OAI cohort. The validation of fully automated technology to assess cartilage volume and its change over time [26] has greatly improved the capacity and reliability of the analysis of the OAI MRI dataset. We examined whether knee pain predicted structural endpoints in people with and without ROA. The OAI intentionally recruited participants at risk of knee OA. This enriched the population for the outcome of interest and thus increased the power of the study to detect significant associations between knee pain and structural outcomes. Knee pain was assessed at baseline and 1 year later using a valid questionnaire, from which knee pain patterns were investigated with a positive linear relationship observed between the frequency of knee pain over 1 year and structural progression over 4 years. This is important because knee pain can fluctuate with time, and thus an isolated baseline assessment may have limited effect on predicting structural progression many years later.

\section{Conclusions}

Greater baseline knee pain, as well as fluctuating and persistent knee pain over 1 year, predicted increased cartilage volume loss and incidence of ROA in people with no ROA and increased cartilage volume loss and progression of ROA in those with ROA over 4 years. With its large cohort, this study provides evidence that knee pain is an important predictor of structural disease progression in population-based individuals with and without knee OA. This study suggests that controlling knee pain early in the disease course as well as over time by targeting the underlying mechanisms may be important for preserving knee structure and reducing the burden of knee OA. Further studies are needed to determine if this is the case.

\section{Additional file}

Additional file 1: Table S1. Associations of knee pain patterns over 2 and 3 years with structural outcomes over 4 years in participants without radiographic knee osteoarthritis at baseline. Table S2. Associations of knee pain patterns over 2 and 3 years with structural outcomes over 4 years in participants with radiographic knee osteoarthritis at baseline. (DOCX $21 \mathrm{~kb})$

\section{Abbreviations}

BMI: Body mass index; K-L: Kellgren-Lawrence; MRI: Magnetic resonance imaging; NNH: Number needed to harm; OA: Osteoarthritis; OAl: Osteoarthritis Initiative; ROA: Radiographic osteoarthritis; VAS: Visual analogue scale;

WOMAC: Western Ontario and McMaster Universities Osteoarthritis Index

\section{Acknowledgements}

We thank the OAl participants and coordinating centre for their work in generating the clinical and radiological data of the OAI cohort and making them publicly available.

\section{Funding}

The OAl is a public-private partnership comprised of five contracts (NO1AR-2-2258, NO1-AR-2-2259, NO1-AR-2-2260, NO1-AR-2-2261, NO1-AR-2-

2262) funded by the National Institutes of Health, a branch of the U.S.

Department of Health and Human Services, in four clinical sites (University of Maryland School of Medicine and The Johns Hopkins University, Baltimore, MD, USA; The Ohio State University, Columbus, OH, USA; University of Pittsburgh, Pittsburgh, PA, USA; and Memorial Hospital of Rhode Island, Pawtucket, RI, USA) and conducted by the OAl study investigators. Private funding partners include Merck Research Laboratories, Novartis Pharmaceuticals Corporation, GlaxoSmithKline, and Pfizer Inc. Private sector funding for the OAl is managed by the Foundation for the National Institutes of Health. For the current study, the image reading was funded by the Osteoarthritis Research Unit, University of Montreal Hospital Research Centre, Montreal, QC, Canada, and the statistical analyses were funded in part by Medibank (Australia). The funders had no role in study design; collection, analysis and interpretation of data; preparation of the manuscript; or the decision to submit the manuscript for publication. YW is a recipient of a National Health and Medical Research Council (NHMRC) Career Development Fellowship (Clinical Level 1, APP1065464). SMH is a recipient of an NHMRC Early Career Fellowship (APP1142198).

\section{Availability of data and materials}

All data generated or analysed during this study are included in this published article and its additional files.

\section{Authors' contributions}

All authors contributed substantially to the conception and design of the work. FA, JPP and JMP contributed to data acquisition. YW and AJT performed statistical analysis and drafted the manuscript. All authors contributed to analysis and interpretation of data for the work and reviewed the manuscript critically for important intellectual content. JMP and MFC had equal contributions as senior authors. All authors had full access to all the data and take responsibility for the integrity of the data and the accuracy of the data analysis. JMP and FMC are the guarantors of the article. All authors read and approved the final manuscript.

\section{Ethics approval and consent to participate}

The OAl study was approved by the Institutional Review Board of the University of California, San Francisco and its affiliates. All participants gave written informed consent.

\section{Consent for publication}

Not applicable.

\section{Competing interests}

YW, AJT, SMH and FMC have no competing interests and are not part of the OAI investigative team. JPP, JMP and FA are not part of the OAI investigative team. JPP and JMP are shareholders in ArthroLab Inc. FA is an employee of ArthroLab Inc

\section{Publisher's Note}

Springer Nature remains neutral with regard to jurisdictional claims in published maps and institutional affiliations.

\section{Author details}

'Department of Epidemiology and Preventive Medicine, School of Public Health and Preventive Medicine, Monash University, 553 St Kilda Road, Melbourne, VIC 3004, Australia. ${ }^{2}$ Medical Imaging Research \& Development, 
ArthroLab Inc., Montreal, QC, Canada. ${ }^{3}$ Osteoarthritis Research Unit, University of Montreal Hospital Research Centre (CRCHUM), Montreal, QC, Canada.

\section{Received: 21 February 2018 Accepted: 22 October 2018 Published online: 06 November 2018}

\section{References}

1. Wluka AE, Wolfe R, Stuckey S, Cicuttini FM. How does tibial cartilage volume relate to symptoms in subjects with knee osteoarthritis? Ann Rheum Dis. 2004;63:264-8

2. Sharma L, Chmiel JS, Almagor O, Dunlop D, Guermazi A, Bathon JM, Eaton $C B$, Hochberg MC, Jackson RD, Kwoh CK, et al. Significance of preradiographic magnetic resonance imaging lesions in persons at increased risk of knee osteoarthritis. Arthritis Rheumatol. 2014;66:1811-9.

3. Urish KL, Keffalas MG, Durkin JR, Miller DJ, Chu CR, Mosher TJ. T2 texture index of cartilage can predict early symptomatic OA progression: data from the osteoarthritis initiative. Osteoarthritis Cartilage. 2013;21:1550-7.

4. Sowers MF, Hayes C, Jamadar D, Capul D, Lachance L, Jannausch M, Welch G. Magnetic resonance-detected subchondral bone marrow and cartilage defect characteristics associated with pain and X-ray-defined knee osteoarthritis. Osteoarthritis Cartilage. 2003;11:387-93.

5. Eckstein F, Cotofana S, Wirth W, Nevitt M, John MR, Dreher D, Frobell R. Greater rates of cartilage loss in painful knees than in pain-free knees after adjustment for radiographic disease stage: data from the osteoarthritis initiative. Arthritis Rheum. 2011;63:2257-67.

6. Miyazaki T, Wada M, Kawahara H, Sato M, Baba H, Shimada S. Dynamic load at baseline can predict radiographic disease progression in medial compartment knee osteoarthritis. Ann Rheum Dis. 2002;61:617-22.

7. Loeser RF, Goldring SR, Scanzello CR, Goldring MB. Osteoarthritis: a disease of the joint as an organ. Arthritis Rheum. 2012;64:1697-707.

8. Wang $X$, Hunter $D, X u J$, Ding C. Metabolic triggered inflammation in osteoarthritis. Osteoarthritis Cartilage. 2015;23:22-30.

9. Neogi T, Guermazi A, Roemer F, Nevitt MC, Scholz J, Arendt-Nielsen L, Woolf C, Niu J, Bradley LA, Quinn E, Frey Law L. Association of joint inflammation with pain sensitization in knee osteoarthritis: the Multicenter Osteoarthritis Study. Arthritis Rheumatol. 2016;68:654-61.

10. Raynauld JP, Martel-Pelletier J, Berthiaume MJ, Labonte F, Beaudoin G, de Guise JA, Bloch DA, Choquette D, Haraoui B, Altman RD, et al. Quantitative magnetic resonance imaging evaluation of knee osteoarthritis progression over two years and correlation with clinical symptoms and radiologic changes. Arthritis Rheum. 2004:50:476-87.

11. Wluka AE, Wolfe R, Davis SR, Stuckey S, Cicuttini FM. Tibial cartilage volume change in healthy postmenopausal women: a longitudinal study. Ann Rheum Dis. 2004;63:444-9.

12. Raynauld JP, Martel-Pelletier J, Berthiaume MJ, Beaudoin G, Choquette D, Haraoui B, Tannenbaum H, Meyer JM, Beary JF, Cline GA, Pelletier JP. Long term evaluation of disease progression through the quantitative magnetic resonance imaging of symptomatic knee osteoarthritis patients: correlation with clinical symptoms and radiographic changes. Arthritis Res Ther. 2006;8:R21.

13. Pelletier JP, Raynauld JP, Berthiaume MJ, Abram F, Choquette D, Haraoui B, Beary JF, Cline GA, Meyer JM, Martel-Pelletier J. Risk factors associated with the loss of cartilage volume on weight-bearing areas in knee osteoarthritis patients assessed by quantitative magnetic resonance imaging: a longitudinal study. Arthritis Res Ther. 2007;9:R74.

14. Saunders J, Ding C, Cicuttini F, Jones G. Radiographic osteoarthritis and pain are independent predictors of knee cartilage loss: a prospective study. Intern Med J. 2012;42:274-80.

15. Spector TD, Dacre JE, Harris PA, Huskisson EC. Radiological progression of osteoarthritis: an 11 year follow up study of the knee. Ann Rheum Dis. 1992; 51:1107-10.

16. Cooper C, Snow S, McAlindon TE, Kellingray S, Stuart B, Coggon D, Dieppe PA. Risk factors for the incidence and progression of radiographic knee osteoarthritis. Arthritis Rheum. 2000;43:995-1000

17. Wolfe F, Lane NE. The longterm outcome of osteoarthritis: rates and predictors of joint space narrowing in symptomatic patients with knee osteoarthritis. J Rheumatol. 2002;29:139-46.

18. Muraki S, Akune T, Oka H, Ishimoto Y, Nagata K, Yoshida M, Tokimura F, Nakamura K, Kawaguchi H, Yoshimura N. Incidence and risk factors for radiographic knee osteoarthritis and knee pain in Japanese men and women: a longitudinal population-based cohort study. Arthritis Rheum. 2012:64:1447-56.

19. Driban JB, Price LL, Eaton CB, Lu B, Lo GH, Lapane KL, McAlindon TE. Individuals with incident accelerated knee osteoarthritis have greater pain than those with common knee osteoarthritis progression: data from the Osteoarthritis Initiative. Clin Rheumatol. 2016;35:1565-71.

20. Peterfy CG, Schneider E, Nevitt M. The osteoarthritis initiative: report on the design rationale for the magnetic resonance imaging protocol for the knee. Osteoarthritis Cartilage. 2008;16:1433-41.

21. Nevitt MC, Peterfy C, Guermazi A, Felson DT, Duryea J, Woodworth T, Chen $\mathrm{H}$, Kwoh K, Harris TB. Longitudinal performance evaluation and validation of fixed-flexion radiography of the knee for detection of joint space loss. Arthritis Rheum. 2007:56:1512-20.

22. Bellamy N, Buchanan WW, Goldsmith CH, Campbell J, Stitt LW. Validation study of WOMAC: a health status instrument for measuring clinically important patient relevant outcomes to antirheumatic drug therapy in patients with osteoarthritis of the hip or knee. J Rheumatol. 1988:15:1833-40.

23. Bellamy N, Bell MJ, Pericak D, Goldsmith CH, Torrance GW, Raynauld JP, Walker V, Tugwell P, Polisson R. BLISS index for analyzing knee osteoarthritis trials data. J Clin Epidemiol. 2007;60:124-32.

24. Ruhdorfer A, Wirth W, Hitzl W, Nevitt M, Eckstein F. Association of thigh muscle strength with knee symptoms and radiographic disease stage of osteoarthritis: data from the osteoarthritis initiative. Arthritis Care Res (Hoboken). 2014;66:1344-53.

25. Martel-Pelletier J, Roubille C, Abram F, Hochberg MC, Dorais M, Delorme P, Raynauld JP, Pelletier JP. First-line analysis of the effects of treatment on progression of structural changes in knee osteoarthritis over 24 months: data from the osteoarthritis initiative progression cohort. Ann Rheum Dis. 2015;74:547-56.

26. Dodin P. Pelletier JP, Martel-Pelletier J, Abram F. Automatic human knee cartilage segmentation from 3D magnetic resonance images. IEEE Trans Biomed Eng. 2010;57:2699-711.

27. Wang Y, Wluka AE, Berry PA, Siew T, Teichtahl AJ, Urquhart DM, Lloyd DG, Jones $\mathrm{G}$, Cicuttini FM. Increase in vastus medialis cross-sectional area is associated with reduced pain, cartilage loss, and joint replacement risk in knee osteoarthritis. Arthritis Rheum. 2012;64:3917-25.

28. Raynauld JP, Pelletier JP, Roubille C, Dorais M, Abram F, Li W, Wang Y, Fairley J, Cicuttini FM, Martel-Pelletier J. Magnetic resonance imagingassessed vastus medialis muscle fat content and risk for knee osteoarthritis progression: relevance from a clinical trial. Arthritis Care Res (Hoboken). 2015;67:1406-15.

29. Teichtahl AJ, Wluka AE, Wang Y, Wijethilake PN, Strauss BJ, Proietto J, Dixon JB, Jones $\mathrm{G}$, Forbes A, Cicuttini FM. Vastus medialis fat infiltration - a modifiable determinant of knee cartilage loss. Osteoarthritis Cartilage. 2015;23:2150-7.

30. Neogi T, Felson D, Niu J, Nevitt M, Lewis CE, Aliabadi P, Sack B, Torner J, Bradley L, Zhang Y. Association between radiographic features of knee osteoarthritis and pain: results from two cohort studies. BMJ. 2009; 339:b2844.

31. Schaible HG, Richter $F$, Ebersberger $A$, Boettger MK, Vanegas $H$, Natura $G$, Vazquez E, Segond von Banchet G. Joint pain. Exp Brain Res. 2009; 196:153-62.

32. Kulkarni B, Bentley DE, Elliott R, Julyan PJ, Boger E, Watson A, Boyle Y, ElDeredy W, Jones AK. Arthritic pain is processed in brain areas concerned with emotions and fear. Arthritis Rheum. 2007;56:1345-54.

33. Valdes AM, De Wilde G, Doherty SA, Lories RJ, Vaughn FL, Laslett LL, Maciewicz RA, Soni A, Hart DJ, Zhang W, et al. The lle585Val TRPV1 variant is involved in risk of painful knee osteoarthritis. Ann Rheum Dis. 2011;70:1556-61.

34. Xu L, Hayashi D, Roemer FW, Felson DT, Guermazi A. Magnetic resonance imaging of subchondral bone marrow lesions in association with osteoarthritis. Semin Arthritis Rheum. 2012;42:105-18.

35. Yusuf E, Kortekaas MC, Watt I, Huizinga TW, Kloppenburg M. Do knee abnormalities visualised on MRI explain knee pain in knee osteoarthritis? A systematic review. Ann Rheum Dis. 2011;70:60-7.

36. Atukorala I, Kwoh CK, Guermazi A, Roemer FW, Boudreau RM, Hannon MJ, Hunter DJ. Synovitis in knee osteoarthritis: a precursor of disease? Ann Rheum Dis. 2016;75:390-5.

37. Eckstein F, Benichou O, Wirth W, Nelson DR, Maschek S, Hudelmaier M, Kwoh CK, Guermazi A, Hunter D. Magnetic resonance imaging-based 
cartilage loss in painful contralateral knees with and without radiographic joint space narrowing: Data from the osteoarthritis initiative. Arthritis Rheum. 2009;61:1218-25.

38. Mundermann A, Dyrby CO, Andriacchi TP. Secondary gait changes in patients with medial compartment knee osteoarthritis: increased load at the ankle, knee, and hip during walking. Arthritis Rheum. 2005;52:2835-44.

39. Jones RK, Chapman GJ, Findlow AH, Forsythe L, Parkes MJ, Sultan J, Felson DT. A new approach to prevention of knee osteoarthritis: reducing medial load in the contralateral knee. J Rheumatol. 2013;40:309-15.

40. Felson DT, Niu J, Guermazi A, Roemer F, Aliabadi P, Clancy M, Torner J, Lewis CE, Nevitt MC. Correlation of the development of knee pain with enlarging bone marrow lesions on magnetic resonance imaging. Arthritis Rheum. 2007:56:2986-92

41. Zhang Y, Nevitt M, Niu J, Lewis C, Torner J, Guermazi A, Roemer F, McCulloch C, Felson DT. Fluctuation of knee pain and changes in bone marrow lesions, effusions, and synovitis on magnetic resonance imaging. Arthritis Rheum. 2011;63:691-9.

42. Felson DT, Chaisson CE, Hill CL, Totterman SM, Gale ME, Skinner KM, Kazis L, Gale DR. The association of bone marrow lesions with pain in knee osteoarthritis. Ann Intern Med. 2001;134:541-9.

43. Hunter DJ, Zhang Y, Niu J, Goggins J, Amin S, LaValley MP, Guermazi A, Genant H, Gale D, Felson DT. Increase in bone marrow lesions associated with cartilage loss: a longitudinal magnetic resonance imaging study of knee osteoarthritis. Arthritis Rheum. 2006;54:1529-35.

44. Tanamas SK, Wluka AE, Pelletier JP, Pelletier JM, Abram F, Berry PA, Wang Y, Jones $\mathrm{G}$, Cicuttini FM. Bone marrow lesions in people with knee osteoarthritis predict progression of disease and joint replacement: a longitudinal study. Rheumatology (Oxford). 2010;49:2413-9.

45. Wang X, Blizzard L, Jin X, Chen Z, Zhu Z, Halliday A, Cicuttini F, Jones G, Ding C. Quantitative assessment of knee effusion-synovitis in older adults: association with knee structural abnormalities. Arthritis Rheumatol. 2016;68: 837-44.

46. Mazzuca SA, Brandt KD, Schauwecker DS, Katz BP, Meyer JM, Lane KA, Bradley JD, Hugenberg ST, Wolfe F, Moreland LW, et al. Severity of joint pain and Kellgren-Lawrence grade at baseline are better predictors of joint space narrowing than bone scintigraphy in obese women with knee osteoarthritis. J Rheumatol. 2005:32:1540-6.

Ready to submit your research? Choose BMC and benefit from:

- fast, convenient online submission

- thorough peer review by experienced researchers in your field

- rapid publication on acceptance

- support for research data, including large and complex data types

- gold Open Access which fosters wider collaboration and increased citations

- maximum visibility for your research: over $100 \mathrm{M}$ website views per year

At $\mathrm{BMC}$, research is always in progress.

Learn more biomedcentral.com/submissions 